J. Lake Sci. (湖泊科学) , 2014, 26(6): 955-962

http://www. jlakes. org. E-mail : jlakes@niglas.ac.cn

(C) 2014 by Journal of Lake Sciences

\title{
鄱阳湖夏季水热通量特征及环境要素影响分析
}

\author{
赵晓松 ${ }^{1}$, 王仕刚 ${ }^{2}$, 李 梅 $^{3}$, 刘元波 $^{1 * *}$ \\ (1: 中国科学院南京地理与湖泊研究所湖泊与环境国家重点实验室,南京 210008) \\ $(2$ : 江西省鄱阳湖水文局, 九江 332800$)$ \\ (3:江西省水文局,南昌 330002$)$
}

摘 要: 气候变化加速了全球水文循环过程, 然而, 气候变化如何影响水体蒸发及其水热通量交换仍然不清楚. 基于浴度相 关系统观测鄱阳湖水体水热通量过程, 在小时和日尺度分析了水热通量的变化规律及其主要影响因子. 研究表明, 潜热通量 日变化波动剧烈, 大部分为正值, 变化范围在 $-50 \sim 580 \mathrm{~W} / \mathrm{m}^{2}$ 之间. 而感热通量数值较小, 变化范围在 $-50 \sim 50 \mathrm{~W} / \mathrm{m}^{2}$ 之 间. 8 月份潜热通量和感热通量均呈波动下降趋势, 均值分别为 167.4 和 $15.9 \mathrm{~W} / \mathrm{m}^{2} .8$ 月份日平均潜热通量和感热通量 之和大于净辐射, 这是由于这一时段储存在水体中的热量释放并补充潜热通量和感热通量. 小时尺度上潜热通量日变化 在相位上与净辐射无显著相关性,而与风速显著相关. 在日尺度变化趋势上, 8 月份日平均潜热通量仍主要受到风速和水 温的影响,感热通量则主要受到风速和饱和水汽压差的影响.

关键词: 潜热通量;感热通量;水面蒸发; 涡度相关; 鄱阳湖

\section{Energy flux measurements and environmental controls in summer over the Poyang Lake, China}

ZHAO Xiaosong ${ }^{1}$, WANG Shigang ${ }^{2}$, LI Mei $^{3} \&$ LIU Yuanbo ${ }^{1}$

(1: State Key Laboratory of Lake Science and Environment, Nanjing Institute of Geography and Limnology, Chinese Academy of Sciences, Nanjing 210008, P. R. China)

(2: Hydrological Bureau of Poyang Lake, Jiangxi Province, Jiujiang 332800, P. R. China)

(3: Hydrological Bureau of Jiangxi Province, Nanchang 330002, P. R. China)

Abstract: Global hydrologic cycles have been intensified by climate change. However, the ways in which climate change affects the evaporation and surface energy budget over inland waters remain unclear. In this study, the surface energy fluxes were measured by an eddy covariance system over the Poyang Lake in August, 2013. We analyzed the hourly and daily variations of energy fluxes, and their responses to the environmental factors. The results showed that the latent heat flux ( LE) has strong turbulence in diurnal variations compared with the sensible heat flux $(\mathrm{H})$ over the Poyang Lake. Most of latent heat fluxes were positive values during the whole day, ranging in $-50-580 \mathrm{~W} / \mathrm{m}^{2}$. The sensible heat flux showed a weakly variance with a range of $-50-50$ $\mathrm{W} / \mathrm{m}^{2}$. The daily mean latent and sensible heat flux showed a decreasing trend with a large fluctuation in August, with the daily mean LE and $\mathrm{H}$ of 167.4 and $15.9 \mathrm{~W} / \mathrm{m}^{2}$, respectively. The sum of daily mean $\mathrm{LE}$ and $\mathrm{H}$ was larger than the net radiation in August, due to heat storage in water body release and supplement of LE and H. In hourly scale, the latent heat flux was out of phase with net radiation, and has a good relationship with the wind speed. In daily scale, the mean LE was mainly controlled by the wind speed and saturate vapor pressure deficit, and $\mathrm{H}$ was controlled by the wind speed and air temperature.

Keywords: Latent heat flux; sensible heat flux; water evaporation; eddy covariance; Poyang Lake

* 国家重点基础研究发展计划“973”项目(2012CB417003)、中国科学院南京地理与湖泊研究所“一三五”战略发展 规划项目 (NIGLAS2012135001) 、江西省水利厅科技项目(KT201121) 和中国科学院 “百人计划”择优支持项目联合 资助. 2013-11-21 收稿;2013-12-26 收修改稿. 赵晓松(1980 ), 女, 博士, 助理研究员; E-mail: xszhao@ niglas. ac. cn.

** 通信作者;E-mail:ybliu@ niglas. ac. cn. 
内陆水体 (湖泊、水库、湿地和河流) 是地球表面的重要组成部分, 是水生动植物的重要栖息地, 同时具 有提供饮用水源和调控洪水等服务功能 ${ }^{[1]}$. 水面蒸发是自然水体水量循环和能量平衡的重要组成. 气候变 暖加速了全球水文循环过程 ${ }^{[2]}$. 然而, 气候变化如何影响内陆水体蒸发及其能量交换仍然不清楚 ${ }^{[3]}$. 由于没 有陆地植被复杂的生态响应过程, 湖泊水体具有快速响应气候变化的特征, 大型内陆湖泊对气候变化具有 指示作用 ${ }^{[4]}$. 因此, 直接测量水体能量交换过程有助于理解气候变化条件下水一大气相互作用变化的物理过 程. 同时, 实际水面蒸发的观测可以为区域大气模型提供验证数据, 从而提高模型对水面蒸发的估算 精度 ${ }^{[5]}$.

基于传统的蒸发血观测的蒸发不能代表实际水面蒸发 ${ }^{[6]}$. 目前, 浴度相关系统作为地表水热通量观测 的国际标准手段,已被广泛应用于地表植被和内陆水体中 ${ }^{[7]}$. 应用浴度相关对内陆水体水热通量交换过程 进行观测, 已有许多研究报道 ${ }^{[4,8-12]}$. 通过对不同湖泊或水库长期或短期的水热通量进行观测, 分析不同时间 尺度水热通量交换过程和对环境因子的响应特征 ${ }^{[9,13-14]}$ 以及湖泊能量平衡过程 ${ }^{[12]}$. 研究表明, 在不同时间 尺度, 潜热通量主要控制因子并不相同 ${ }^{[15]}$. Liu 等 ${ }^{[14]}$ 对比了不同纬度湖泊水热通量特征, 发现低纬度的浅水 湖泊与高纬度的深水湖泊相比, 其热储存能力相对较小, 对气象条件的响应速度则更快. Venäläinen 等 $^{\left[{ }^{[6]}\right.}$ 认 为潜热通量还受到水体面积的影响, 大小不同的湖泊水体潜热通量对环境因子具有不同的响应 ${ }^{[17]}$. 目前, 应 用浴度相关系统对水体水热通量的研究, 除了加拿大 Great Slave 湖和 Great Bear 湖为面积大于 $25000 \mathrm{~km}^{2}$ 的 大型湖泊 ${ }^{[9]}$, 大多数针对面积小于 $150 \mathrm{~km}^{2}$ 的中小湖泊 ${ }^{[8,11]}$, 且多位于高纬度地区, 而缺少中低纬度地区大 型湖泊水热通量特征的研究,其环境因子影响机制仍不清楚.

鄱阳湖是我国最大的淡水湖泊, 位于长江中下游地区, 其最大水面面积达到 4000 余 $\mathrm{km}^{2}$, 为研究大型湖 泊水体蒸发提供了天然条件. 本研究基于浴度相关系统观测鄱阳湖水体的水热通量, 分析鄱阳湖水体水热 通量的变化特征, 并分析不同时间尺度影响其变化的主要环境因子, 可增加对中纬度大型湖泊水体水热通 量特征的认识,并对内陆水资源管理和水文循环具有重要意义.

\section{1 研究方法}

\section{1 研究站点与观测仪器}

研究站点位于鄱阳湖东部, 都昌县附近的蛇山岛上 $\left(29^{\circ} 5^{\prime} \mathrm{N}, 116^{\circ} 24^{\prime} \mathrm{E}\right.$; 图 1). 鄱阳湖面积为 $4380 \mathrm{~km}^{2}$ (2010 年鄱阳湖基础地理信息测量), 年内水位变化剧烈, 平均变幅达 $11 \mathrm{~m}$ 左右 ${ }^{[18]}$. 鄱阳湖地属亚热带湿润 季风气候, 湖区年平均气温为 $17.1^{\circ} \mathrm{C}$, 多年平均降水量为 $1570 \mathrm{~mm}$, 降水集中在 $4-6$ 月, 占全年降水的 $45 \% \sim 50 \%$. 主风向为北和北偏西风. 西北方向距离岸边约 $6 \mathrm{~km}$, 距离棠荫岛 $2 \mathrm{~km}$. 蛇山岛的面积约为 0.2 $\mathrm{km}^{2}$, 南北长 $500 \mathrm{~m}$, 东西长 $450 \mathrm{~m}$.

为减少蛇山岛本身对观测的影响, 观测铁搭架设在蛇山岛北部, 浴度相关系统仪器安装在 $38 \mathrm{~m}$ 的观测 铁塔上. 考虑到岛本身的高度, 观测高度距离湖面 $58 \mathrm{~m}$. 采用三维超声风速和 $\mathrm{CO}_{2} / \mathrm{H}_{2} \mathrm{O}$ 分析仪 ( EC150, Campbell Scientific Inc., Logan, UT, USA) 测量三维风速和大气的 $\mathrm{H}_{2} \mathrm{O}$ 浓度, 采用频率为 $10 \mathrm{~Hz}$, 通过数据采集 器( CR3000, Campbell Inc., ) 进行存储. 用净辐射仪 (CNR4, Kipp \& Zonen B. V., Delft, The Netherlands) 观测辐 射平衡的 4 个组分 (向下、向上长波辐射, 向下、向上短波辐射). 采用小气候系统仪器观测气温和相对湿度 (HMP155A, Vaisala Helsinki, Fenland). 浴度相关系统和小气候系统安装高度为 $38 \mathrm{~m}$, 辐射观测安装高度为 $2.5 \mathrm{~m}$. 水热通量、辐射和小气候系统数据的输出步长为 $30 \mathrm{~min}$. 用水温计 (SWL1-1) 测量 $50 \mathrm{~cm}$ 水深处的水 温. 应用 E601-B 蒸发血测量蒸发, 地点位于棠荫岛气象观测站内. 水温和蒸发血蒸发的采样频率为每天一 次, 于上午 8 点采集数据. 浴度相关系统仪器和小气候仪器于 2013 年 7 月 26 日安装并开始观测. 本文选取 2013 年 8 月 1 日一- 8 月 30 日数据进行分析,避免水位降低后洲滩出露的影响,通量信息主要来自于鄱阳湖 水体. 测量期间风向以北偏西及南偏东为主.

\section{2 数据处理与分析}

对浴度相关观测的 $10 \mathrm{~Hz}$ 数据进行笁选和修正. 数据处理采用目前普遍使用较为成熟的方法, 应用 EddyPro 软件进行处理, 包括: (1) 数据篮选: 通过信号强度和诊断值判断数据有效性, 应用 4 倍标准差剔除野 点; (2) 应用平面坐标拟合方法进行倾斜修正 ${ }^{[19]} ;$; (3) 频率损失修正 ${ }^{[20]}$; (4) 感热的超声虚温修正 ${ }^{[21]}$; 


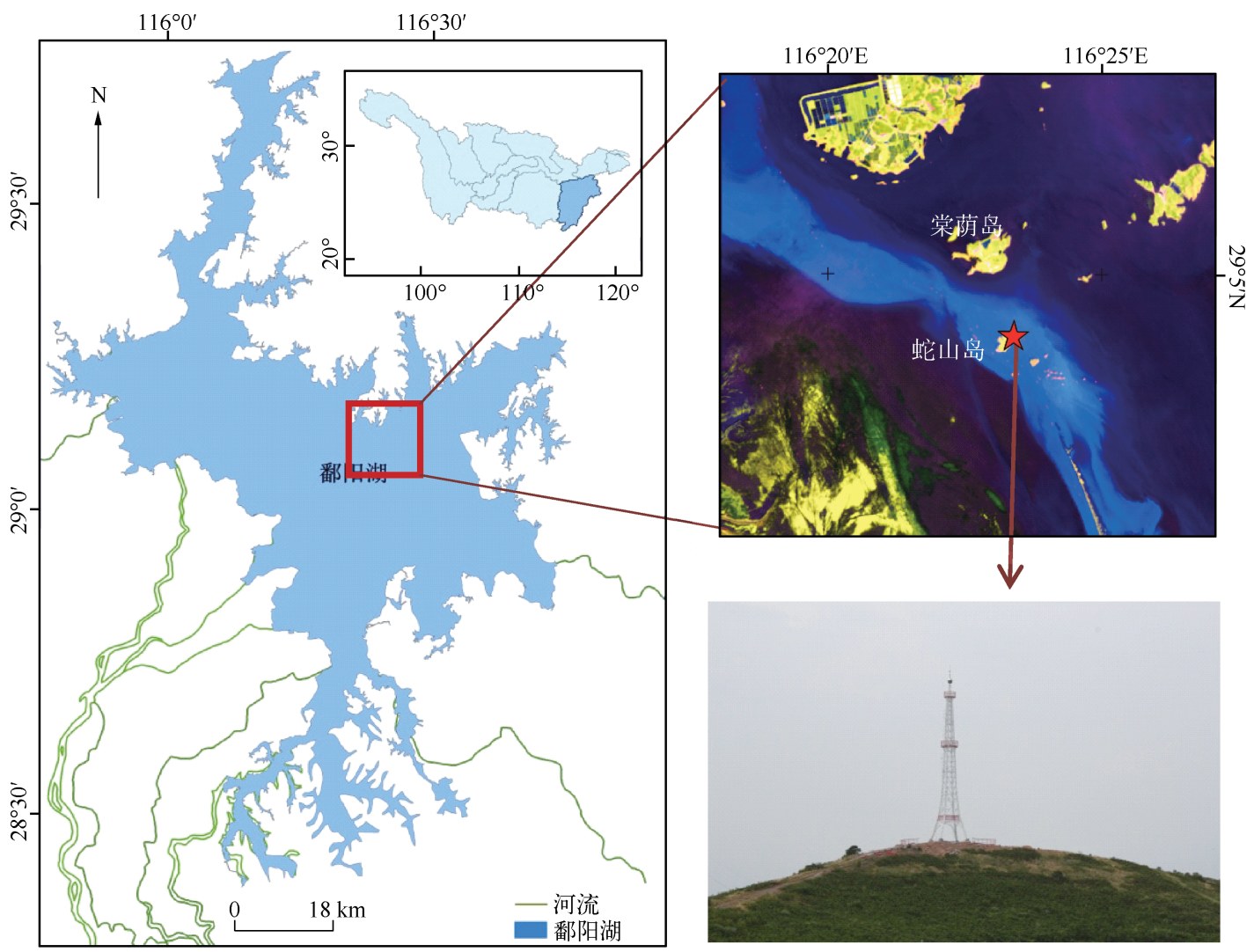

图 1 鄱阳湖及蛇山岛观测地点位置

Fig. 1 The map of the Poyang Lake and measurement site at Sheshan Island

(5) 密度效应修正: WPL 修正 ${ }^{[22]}$. 通过湍流谱分析和平稳性检验和总体湍流特征检验进行数据质量控 制 ${ }^{[23]}$. 获得 3 个评价等级的通量数据, 分别为 0 (高质量)、1 (中质量)、2(低质量) 数据.

进行数据处理和评价后,输出 $30 \mathrm{~min}$ 平均的通量数据,用于进一步的分析. 在计算日蒸散量时, 由于观 测野点和数据质量等问题造成通量数据缺失, 需要对缺失的数据进行插补, 本研究所选时段数据缺失比例 为 $9.5 \%$, 当连续缺失数据小于 3 个时, 应用线性内插方法进行数据插补 ${ }^{[24]}$.

通过数据质量控制, 选取高质量和中质量的数据 (数据质量 $<2$ ) 进行环境因子分析. 本文选择非 参数检验方法中的 Kendall 秩相关分析进行. 常用的 Pearson 线性相关分析属于参数检验方法, 它需要 满足数据符合正态分布的假设. 而分析数据中如风速、相对湿度、潜热和感热通量等并不能完全满足 这一假设. 而 Kendall 秩相关分析方法是一种非参数性质 (与分布无关) 的秩统计方法, 不受样本数据 分布形态的影响. 应用 SPSS 软件中 Kendall 秩相关方法实现不同时间尺度环境因子对潜热和感热通量 的相关分析.

浴度相关系统观测的通量代表了上风向一定范围内的通量信息, 即通量源区 (Footprint). 观测塔所观测 的通量数据表示的是通量贡献区内的平均状况. 对于不均匀的下垫面条件,常需利用 Footprint 分析了解下 垫面不同位置,特别是感兴趣的下垫面, 对传感器所测湍流通量贡献的大小及对观测数据做质量判别. 关于 通量贡献区的计算有很多解析模型 ${ }^{[25-27]}$, 本研究应用 Hsieh 等 ${ }^{[26]}$ 的方法分析不同大气条件下的 Footprint 分 布,来确定观测时段内通量信息来源. 通量贡献区分布如图 2 所示. 2013 年 8 月 1 日- 8 月 30 日期间风向主 要来自西北和东南方向. 在稳定大气条件下, $90 \%$ 的通量信息来自于上风向距观测塔 $5 \mathrm{~km}$ 范围内. 在不稳定 大气条件下,通量贡献区的范围较小, 在距观测塔 $1 \sim 2 \mathrm{~km}$ 范围内. 观测期内主要通量信息均来自于水体,受 
蛇山岛和棠荫岛的影响较小.
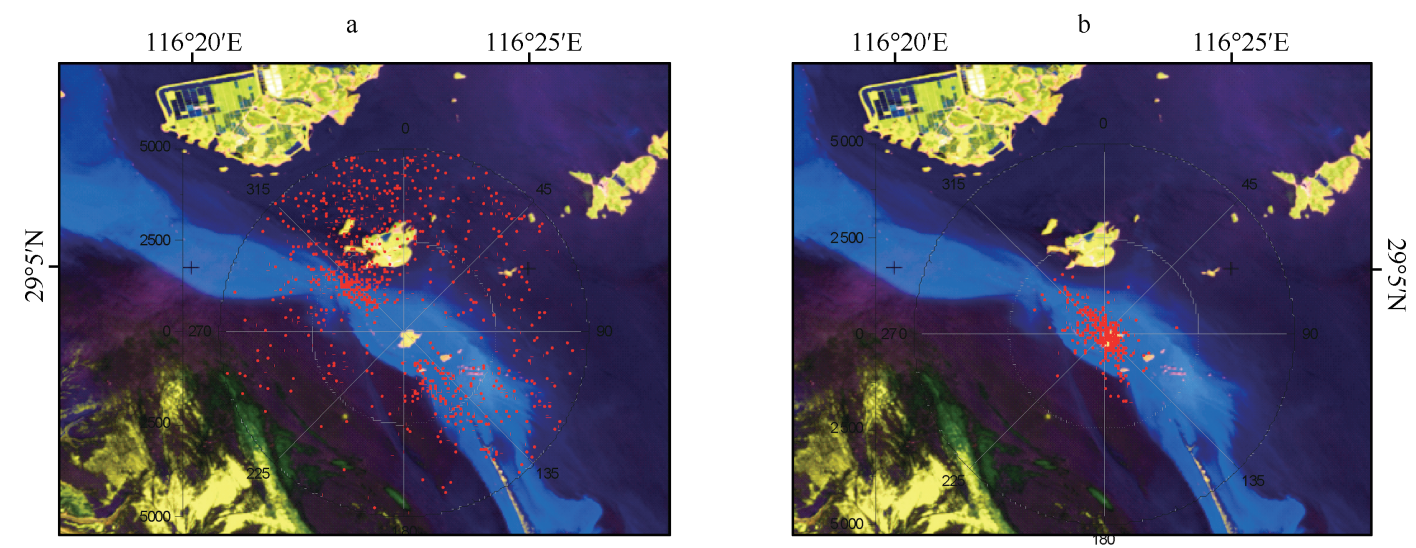

图 2 源区 Footprint 分布图:稳定条件(a)、不稳定条件 (b) $\left(0^{\circ}\right.$ 表示北向, $90^{\circ}$ 表示东向， $180^{\circ}$ 表示南向, $270^{\circ}$ 表示西向; 底图为 Landsat 8 6-5-4 波段合成影像, 时间为 2013 年 8 月 2 日,水位 $15.6 \mathrm{~m}$ )

Fig. 2 The distribution of Footprint for energy fluxes observation around the Sheshan Island: over stable atmospheric condition(a) and unstable atmospheric condition(b)

\section{2 研究结果}

\section{1 湖泊水热通量的小时尺度变化特征}

基于浴度相关观测的 $30 \mathrm{~min}$ 平均通量数据,选取 8 月 6 日- 8 月 8 日时间段,分析其日变化过程(图 3). 净辐射、潜热通量和感热通量是能量平衡中的 3 个重要参数, 所选时段内, 净辐射的日变化过程呈现单峰正 态分布规律, 为典型的晴天天气. 净辐射白天为正值, 最大值 $660 \mathrm{~W} / \mathrm{m}^{2}$ 在 $12: 00$ 左右取得, 夜间为负值, 平均 为 $-50 \mathrm{~W} / \mathrm{m}^{2}$. 不同于陆地植被下垫面的日变化趋势 ${ }^{[28]}$, 鄱阳湖水体潜热通量无明显的规律性日变化过程. 潜热通量日变化波动剧烈, 变化范围在 $-50 \sim 580 \mathrm{~W} / \mathrm{m}^{2}$ 之间. 2013 年 8 月 6 日- 8 月 8 日期间, 潜热通量在 $0: 00-12: 00$ 之间保持较高的值, $12: 00$ 左右出现一个低值, 随后潜热通量呈现先升高又逐渐下降的趋势. 潜 热通量以正值为主, 只在夜间出现个别的负值, 其与净辐射的日变化过程存在显著差异.气温和饱和水汽压 差 (VPD) 表现为与净辐射相似的变化趋势, 其峰值与净辐射存在 $3 \mathrm{~h}$ 的时间延迟. 气温和 VPD 的最小值出 现在早上 $7: 00$, 而最大值出现在 $17: 00$ 左右. 气温日变化幅度较小, 在 $30 \sim 35^{\circ} \mathrm{C}$ 之间, $\mathrm{VPD}$ 变化范围为 $0.8 \sim$ $3.4 \mathrm{kPa}$. 潜热通量与气温和 VPD 的日变化也存在差异, 但与风速的变化具有很好的一致性. 对应风速的变 化过程, 当风速降低时, 潜热通量出现明显的低值, 而风速高时则对应潜热通量的峰值. 但同时还发现在 8 月 8 日 15:00 潜热通量出现一个峰值, 此时风速与 6:00 左右的风速相当, 而气温和 VPD 达到高值, 使得潜 热通量较前期明显提高, 达到新的高值点. 这说明在风速一定的条件下, 较高的气温和 VPD 有利于潜热通量 交换过程. 感热通量日变化较小, 范围在 $-50 \sim 50 \mathrm{~W} / \mathrm{m}^{2}$ 之间. 感热通量表现为弱的单峰日变化过程, 白天为 正值, 最大值出现在 $12: 00$ 前后, 夜间为负值, 变化范围较小. 由于水体具有很强的热储存能力, 水温和气温 日变幅不大, 因此水体下垫面的感热通量通常较小 ${ }^{[12]}$. 从变化趋势上, 感热通量与净辐射存在一定的相关 性. 对于水体下垫面潜热和感热通量这种剧烈波动的日变化过程, 其他湖泊和水库也有相似的报道 ${ }^{[8,12,16]}$.

\section{2 湖泊水热通量的日尺度变化特征}

8 月鄱阳湖日平均通量及环境因子的变化过程如图 4 所示. 净辐射呈波动下降趋势, 变化范围在 $47.4 \sim$ $179.4 \mathrm{~W} / \mathrm{m}^{2}$ 之间, 均值为 $136.1 \mathrm{~W} / \mathrm{m}^{2}$. 潜热通量也呈下降趋势, 且波动较为剧烈, 变化范围在 $75.0 \sim$ $326.1 \mathrm{~W} / \mathrm{m}^{2}$ 之间, 均值为 $167.4 \mathrm{~W} / \mathrm{m}^{2}$. 感热通量变化幅度较小, 范围在 $-18.7 \sim 54.5 \mathrm{~W} / \mathrm{m}^{2}$ 之间, 均值为 15.9 $\mathrm{W} / \mathrm{m}^{2}$. 从 8 月日平均能量通量变化可以看出, 潜热通量和感热通量之和大于净辐射. Liu 等 ${ }^{[14]}$ 在美国水库也 
发现了这一现象,通常发生在 9 月至次年 2 月. 这是由于该时期水温呈下降趋势, 储存在 水体中的热量向外释放, 并补充潜热通量和 感热通量 ${ }^{[29]} .8$ 月鄱阳湖日平均气温的变化 趋势与净辐射相似, 呈下降趋势, 变化范围为 $26.8 \sim 33.1^{\circ} \mathrm{C}$, 均值为 $30.9^{\circ} \mathrm{C}$. 水温变化表 现为下降趋势, 8 月 24 日之后又有所回升, 变化范围在 $27.4 \sim 32.0^{\circ} \mathrm{C}$ 之间, 均值为 $30.2^{\circ} \mathrm{C}$. 由于水温为早上 $8: 00$ 的水体温度, 不能代表全天平均水温, 因此, 无法直接与日 均气温进行比较. 通常在 6-10 月份, 日平均 气温大于水温 ${ }^{[14]}$. 相对湿度和 VPD 呈相反 的变化过程, 在 8 月 15 日之后, 受阴天和降 水的影响, 相对湿度有所增加, 而 VPD 呈现 下降的趋势, 其均值分别为 $70.0 \%$ 和 1.39 $\mathrm{kPa} .8$ 月份风速均值为 $5.1 \mathrm{~m} / \mathrm{s}, 8$ 月中旬风 速下降. 受到天气过程的影响, 8 月 22 日出 现最大的日均风速 $(13.4 \mathrm{~m} / \mathrm{s}) .8$ 月鄱阳湖 水位呈持续下降趋势, 水位从月初的 $15.7 \mathrm{~m}$ 降到 8 月 24 日的 $13.7 \mathrm{~m}$, 随后受到降水的影 响,水位略有回升,至月末达到 $13.9 \mathrm{~m}$.

\section{3 环境因子的影响}

无论水面蒸发还是陆面蒸发, 都受到多 种气候因子的综合影响. 气温、湿度、饱和水 汽压差、太阳辐射、风速、气温日较差等都会
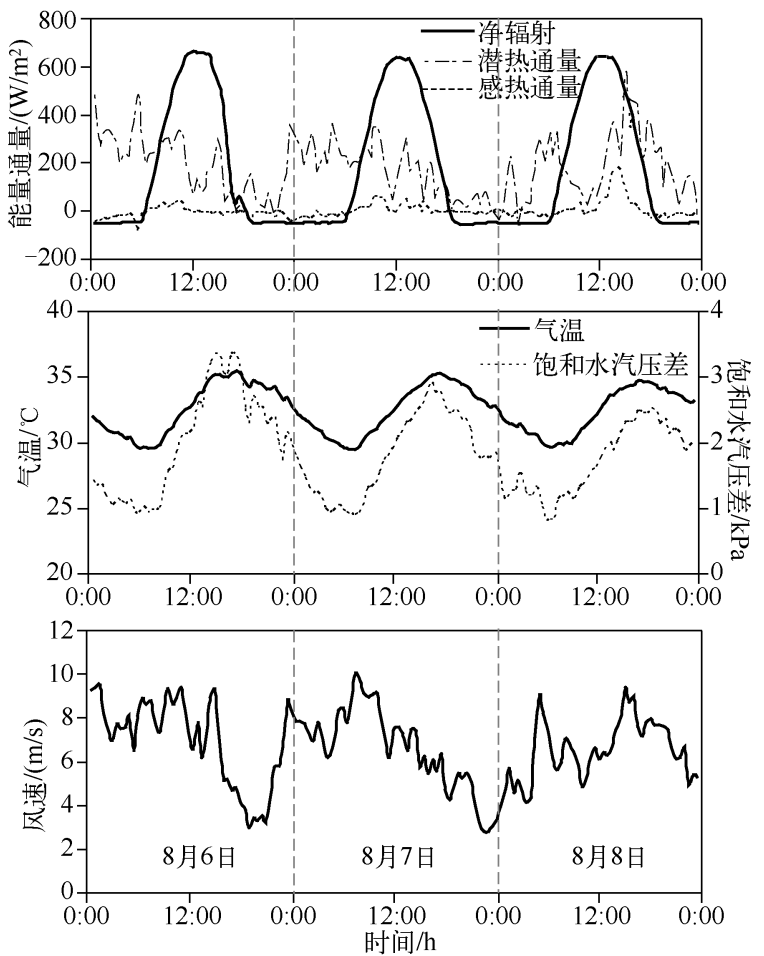

图 32013 年 8 月 6 日一- 8 月 8 日水热通量及净辐射、 气象要素日变化过程

Fig. 3 Diurnal variation of energy fluxes, net radiation and meteorological factors during Aug. 6 to Aug. 8,2013 对蒸发量造成影响 ${ }^{[12]}$. Kendall 秩相关分析 显示, 在小时尺度, $30 \mathrm{~min}$ 平均潜热通量与风速的相关性最显著 $(r=0.337)$, 其次是净辐射 $(r=0.168)$ 、饱 和水汽压差 $(r=0.100)$ 和相对湿度 $(r=-0.123)$, 均通过 $99 \%$ 置信区间检验, 而与气温无显著相关性. 感热 通量则表现为与净辐射相关性最大 $(r=0.425)$, 其次为风速 $(r=-0.234)$, 并通过 $99 \%$ 置信区间检验 (表 1). 尽管统计表明感热通量与净辐射呈显著相关,但通过两者的对比发现,其离散程度非常大, 从日变化过 程 (图 3) 可以发现, 当夜间净辐射很小时, 潜热通量值或很大, 或很小. 因此, 在日变化过程中潜热通量与净 辐射无显著相关. 感热通量体现了温度的变化过程, 主要受到辐射的驱动. 相关研究也表明, 在小时尺度上, 风速是潜热通量的主要控制因子, 与净辐射无显著相关, 说明近地面平均风速对水面蒸发的影响主要是通 过湍流交换作用实现的 ${ }^{[34]}$. 日尺度湍流交换主要是动量驱动,而不是能量驱动的.

表 1 潜热通量和感热通量 (30 min 平均) 与环境因子的 Kendall 秩相关系数 $(n=1346)$

Tab. 1 The Kendall rank coefficient within 30 min mean energy fluxes and controlling factors(net radiation, air temperature, wind speed, saturate vapor pressure deficit, relative humid $)(n=1346)$

\begin{tabular}{cccccc}
\hline & 净辐射 & 气温 & 风速 & 饱和水气压差 & 相对湿度 \\
\hline 潜热通量 & $0.168^{* *}$ & 0.010 & $0.337^{* *}$ & $0.100^{* *}$ & $-0.123^{* *}$ \\
感热通量 & $0.425^{* *}$ & 0.009 & $-0.234^{* *}$ & 0.003 & -0.009 \\
\hline
\end{tabular}

* 表示通过 $95 \%$ 置信区间检验; ** 表示通过 $99 \%$ 置信区间检验.

水体潜热和感热通量是多个因素综合作用的结果. 考虑风速 $(W S)$ 、气温 $(T a)$ 、相对湿度 $(R H)$ 、饱和水 汽压差 $(V P D)$ 和净辐射 $(R n)$ 等因素, 通过逐步回归方法分析潜热通量 $(L E)$ 和感热通量 $(H)$ 的关系, 得到潜 

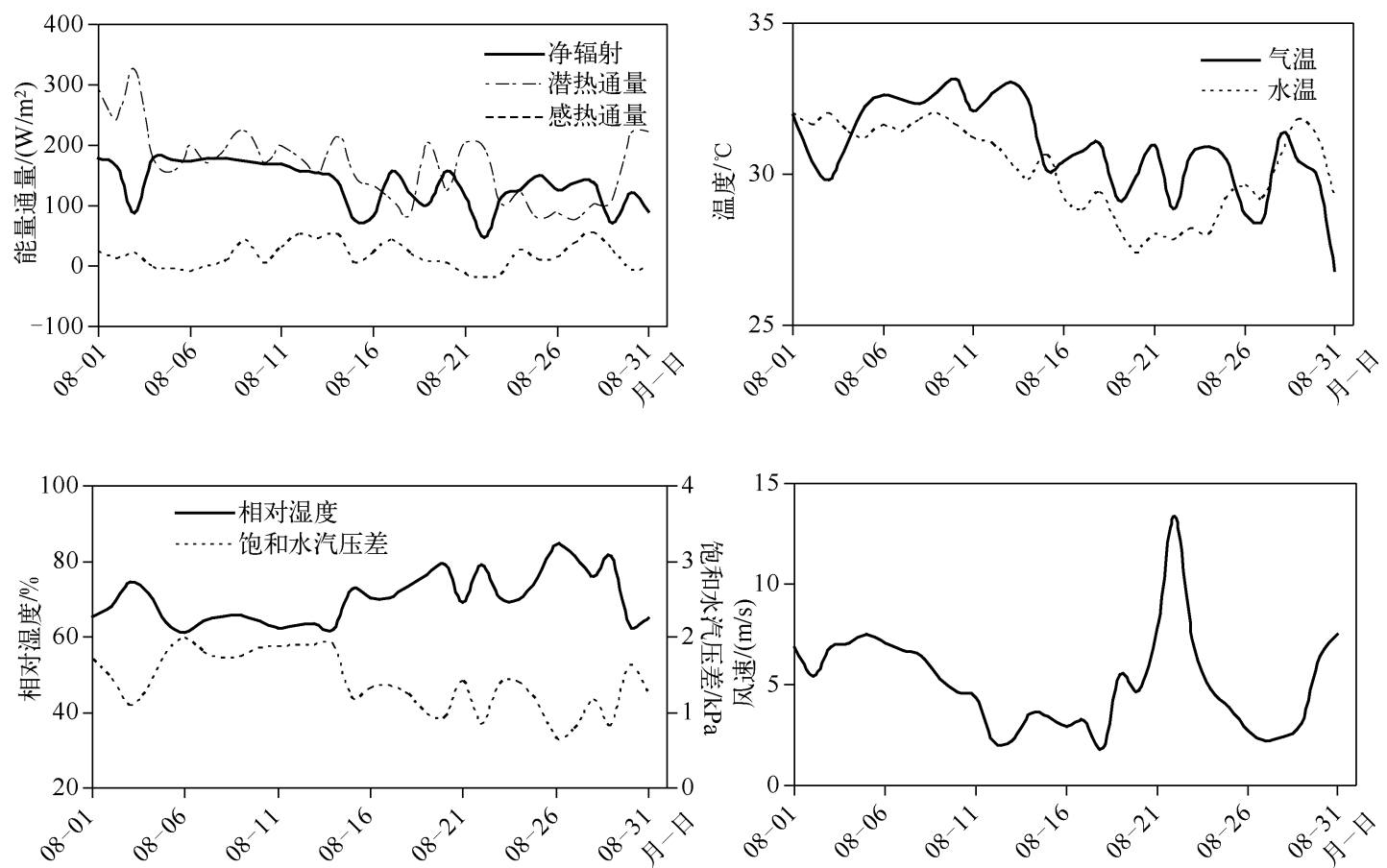

图 42013 年 8 月份鄱阳湖日平均水热通量及净辐射、气象水文要素变化过程

Fig. 4 Variation of daily mean energy flux and meteorological factors over the Poyang Lake in August,2013

热通量的回归方程为 $L E=13.81 \mathrm{WS}-6.67 R H-70.60 \mathrm{VPD}+657.37$ (相关系数 $R^{2}=0.21$, 均方根误差 $R M S E=$ $103.46 \mathrm{~W} / \mathrm{m}^{2}$ ), 风速、相对湿度和饱和水汽压差通过检验进人回归方程. 在小时尺度上, 方程仅能解释潜热 通量变化的 $21 \%$. 感热通量的回归方程为 $H=0.132 R n-4.082 \mathrm{WS}+17.144\left(R^{2}=0.37, R M S E=43.6 \mathrm{~W} / \mathrm{m}^{2}\right)$, 净辐射和风速通过检验进人回归方程,方程仅能解释感热通量变化的 $37 \%$.

Kendall 秩相关分析显示, 日平均潜热通量仍然与风速的相关性最显著 $(r=0.372)$, 通过 $99 \%$ 置信区间 检验, 其次是相对湿度 $(r=-0.351)$ 和水温 $(r=0.345)$, 而与辐射和气温无显著相关性. 感热通量则表现为 与风速相关性最显著 $(r=-0.557)$, 通过 $99 \%$ 置信区间检验, 而与其他因子无显著相关 (表 2). 通过逐步回 归方法, 得到日平均潜热通量与环境因子相关关系的回归方程 $L E=9.948 W S+16.96 T w-15.08 D T-2.75$ $R H-128.27\left(R^{2}=0.628, R M S E=40.67 \mathrm{~W} / \mathrm{m}^{2}\right)$, 风速、水温、相对湿度和气温日较差通过检验进人回归方 程. 在日尺度上, 方程能解释潜热通量变化的 $63 \%$. 感热通量的回归方程为 $H=10.358-4.69 \mathrm{WS}+$ $18.88 \mathrm{VPD}\left(R^{2}=0.45, R M S E=15.13 \mathrm{~W} / \mathrm{m}^{2}\right)$, 风速和饱和水汽压差通过检验进人回归方程, 方程能解释感热 通量变化的 $45 \%$.

表 2 日平均潜热和感热通量与环境因子的 Kendall 秩相关系数 $(n=31)$

Tab. 2 The Kendall rank coefficient within daily mean energy fluxes and controlling factors (net radiation, air temperature, wind speed, saturate vapor pressure deficit, relative humid, water temperature at $50 \mathrm{~cm}$ depth, and differ of maximum and minimum air temperature $)(n=31)$

\begin{tabular}{rccccccc}
\hline & 净辐射 & 气温 & 风速 & 饱和水汽压差 & 相对湿度 & 水温 & 气温日较差 \\
\hline 潜热通量 & 0.118 & 0.088 & $0.372^{* *}$ & $0.290^{*}$ & $-0.351^{* *}$ & $0.345^{*}$ & -0.234 \\
感热通量 & 0.049 & 0.191 & $-0.557^{* *}$ & 0.067 & -0.006 & 0.134 & 0.067 \\
\hline
\end{tabular}

* 表示通过 $95 \%$ 置信区间检验; $* *$ 表示通过 $99 \%$ 置信区间检验. 
针对湖泊、水库等水体水热通量的研究, Assouline 等 $^{[8]}$ 在以色列中纬度 Eshkol 水库报道了相似的日变 化过程, 研究表明潜热通量的日变化相位上与净辐射无关, 而与风速相关, 感热通量与水、气温差相关性显 著. 本研究也得到相似的结果. 在季节变化上, Lenters 等 ${ }^{[15]}$ 认为潜热通量季节变化受到辐射和温度驱动, 与 风速无关. 年际间变化则主要受到辐射影响, 其次是温度或湿度. Nordbo 等 ${ }^{[12]}$ 认为水一气温差是影响感热通 量的重要因素, 而潜热通量主要受饱和水汽压差的影响. 太阳辐射对蒸发的影响具有清晰的物理机制, 长期 以来许多学者应用总辐射或净辐射资料计算蒸发潜力和蒸发量 ${ }^{[30-32]}$. 本研究季节变化趋势上, 潜热通量仍 然主要受到风速和饱和水汽压差等的影响, 而未表现出与辐射和温度的相关性, 这可能是因为本研究仅为 一个月时间周期,辐射和温度的变化幅度较小,并未表现出长期变化趋势.

\section{3 结论}

湖泊水热通量是水量平衡要素的重要组成部分. 本研究基于浴度相关系统观测鄱阳湖水体水热通量, 分析了水热通量在小时和日尺度的变化规律及其主要影响因子. 研究表明,潜热通量日变化波动剧烈且变 化范围大,而感热通量变化范围较小. 8 月份日平均潜热通量和感热通量均呈波动下降趋势, 潜热通量和感 热通量之和大于净辐射. 潜热通量的日变化过程与净辐射没有显著相关性, 说明净辐射不是潜热通量日变 化的直接驱动因子. 通过多元回归分析, 在小时尺度, 潜热通量主要受风速、相对湿度和饱和水汽压差的影 响,方程能解释潜热通量变化的 $21 \%$. 而感热通量受到净辐射和风速的影响, 方程能解释感热通量变化的 $37 \%$. 在日尺度, 日平均潜热通量主要受风速、水温、相对湿度和气温日较差等综合影响,方程能解释潜热通 量变化的 $63 \%$. 感热通量受风速和饱和水汽压差的影响,方程能解释感热通量变化的 $45 \%$. 湖泊水体蒸发对 了解湖泊水量变化及区域水循环和水资源具有重要意义.

致谢: 感谢鄱阳湖水文局提供蛇山岛水文数据. 感谢蛇山岛基地工作人员在野外仪器维护等给予的帮助. 感 谢江西省水文局洪全祥处长、江西省鄱阳湖水文局龚向民副调研员、中国科学院南京地理与湖泊研究所冯 徽徽博士等对仪器安装和维护给予的帮助。

\section{4 参考文献}

[ 1 ] Assel RA, Quinn FH, Sellinger CE. Hydroclimatic factors of the recent record drop in Laurentian Great Lakes water levels. Bulletin of the American Meteorological Society, 2004, 85 (8) : 1143-1151.

[ 2 ] Huntington TG. Evidence for intensification of the global water cycle: review and synthesis. Journal of Hydrology, 2006, $319(1): 83-95$.

[ 3 ] Zhang Q, Liu H. Interannual variability in the surface energy budget and evaporation over a large southern inland water in the United States. Journal of Geophysical Research : Atmospheres, 2013, 118(10) : 4290-4302.

[ 4 ] Blanken PD, Rouse WR, Culf AD et al. Eddy covariance measurements of evaporation from Great Slave Lake, Northwest Territories, Canada. Water Resources Research, 2000, 36(4) : 1069-1077.

[ 5 ] Long Z, Perrie W, Gyakum J et al. Northern lake impacts on local seasonal climate. Journal of Hydrometeorology, 2007, $\mathbf{8}(4): 881-896$.

[ 6 ] Grismer M, Orang M, Snyder R et al. Pan evaporation to reference evapotranspiration conversion methods. Journal of Irrigation and Drainage Engineering, 2002, 28(3) : 180-184.

[ 7 ] Baldocchi DD. Assessing the eddy covariance technique for evaluating carbon dioxide exchange rates of ecosystems: past, present and future. Global Change Biology, 2003, (4) : 479-492.

[ 8 ] Assouline S, Tyler S, Tanny J et al. Evaporation from three water bodies of different sizes and climates: Measurements and scaling analysis. Advances in Water Resources, 2008, 31(1) : 160-172.

[ 9 ] Rouse WR, Blanken PD, Bussières N et al. An investigation of the thermal and energy balance regimes of Great Slave and Great Bear Lakes. Journal of Hydrometeorology, 2008, (6) : 1318-1333.

[10] Tanny J, Cohen S, Assouline S et al. Evaporation from a small water reservoir: Direct measurements and estimates. Journal of Hydrology, 2008, 51 ( 1 ) : 218-229.

[11] Liu H, Zhang Y, Liu S et al. Eddy covariance measurements of surface energy budget and evaporation in a cool season over southern open water in Mississippi. Journal of Geophysical Research, 2009, 114( D4) : D04110. 
[12] Nordbo A, Launiainen S, Mammarella I et al. Long-term energy flux measurements and energy balance over a small boreal lake using eddy covariance technique. Journal of Geophysical Research : Atmospheres, 2011, 116(D2) : D02119.

[13] Schertzer WM, Rouse WR, Blanken PD et al. Over-lake meteorology and estimated bulk heat exchange of Great Slave Lake in 1998 and 1999. Journal of Hydrometeorology, 2003, 4(4) : 649-659.

[14] Liu H, Zhang Q, Dowler G. Environmental controls on the surface energy budget over a large southern inland water in the United States: An analysis of one-year eddy covariance flux data. Journal of Hydrometeorology, 2012 ,13 (6) : 1893-1910.

[15] Lenters JD, Kratz TK, Bowser CJ. Effects of climate variability on lake evaporation: Results from a long-term energy budget study of Sparkling Lake, northern Wisconsin (USA). Journal of Hydrology, 2005, 308(1) : 168-195.

[16] Venäläinen A, Frech M, Heikinheimo M et al. Comparison of latent and sensible heat fluxes over boreal lakes with concurrent fluxes over a forest: Implications for regional averaging. Agricultural and Forest Meteorology, 1999, 98 : 535-546.

[17] Spence C, Rouse WR, Worth D et al. Energy budget processes of a small northern lake. Journal of Hydrometeorology, 2003, 4(4): 694-701.

[18] 谢冬明,郑 鹏,邓红兵等. 鄱阳湖湿地水位变化的景观响应. 生态学报,2011,31(5):1269-1276.

[19] Wilczak JM, Oncley SP, Stage SA. Sonic anemometer tilt correction algorithms. Boundary-Layer Meteorology, 2001,99 (1) : 127-150.

[20] Moore C. Frequency response corrections for eddy correlation systems. Boundary-Layer Meteorology, 1986, 37 (1/2) : $17-35$.

[21] Van Dijk AI. Estimates of $\mathrm{CO}_{2}$ uptake and release among European forests based on eddy covariance data. Global Change Biology, 2004, 10(9): 1445-1459.

[22] Webb EK, Pearman GI, Leuning R. Correction of flux measurements for density effects due to heat and water vapour transfer. Quarterly Journal of the Royal Meteorological Society, 1980, 106(447) : 85-100.

[23] Foken T, Göockede M, Mauder M et al. Post-field data quality control, in Handbook of micrometeorology. Dordrecht: Springer, 2005 : 181-208.

[24] Falge E, Baldocchi D, Olson R et al. Gap filling strategies for defensible annual sums of net ecosystem exchange. Agricultural and Forest Meteorology, 2001, 107(1): 43-69.

[25] Haenel HD, Grünhage L. Footprint analysis : a closed analytical solution based on height-dependent profiles of wind speed and eddy viscosity. Boundary-Layer Meteorology, 1999, 93(3) : 395-409.

[26] Hsieh CI, Katul G, Chi TW. An approximate analytical model for footprint estimation of scalar fluxes in thermally stratified atmospheric flows. Advances in Water Resources, 2000, 23(7): 765-772.

[27] Schmid HP. Footprint modeling for vegetation atmosphere exchange studies: a review and perspective. Agricultural and Forest Meteorology, 2002, 113(1): 159-183.

[28] Eaton AK, Rouse WR, Lafleur PM et al. Surface energy balance of the western and central Canadian subarctic: Variations in the energy balance among five major terrain types. Journal of Climate, 2001, 14(17) : 3692-3703.

[29] Rouse WR, Oswald CJ, Binyamin J et al. The role of northern lakes in a regional energy balance. Journal of Hydrometeorology, 2005, 6(3) : 291-305.

[30 ] Rosenberry DO, Winter TC, Buso DC et al. Comparison of 15 evaporation methods applied to a small mountain lake in the northeastern USA. Journal of Hydrology, 2007, 340 (3) : 149-166.

[31] Brutsaert W. Evaporation into the atmosphere: theory, history, and applications. Dordrecht: Reidel, 1982.

[32 ] De Bruin H, Keijman J. The Priestley-Taylor evaporation model applied to a large, shallow lake in the Netherlands. Journal of Applied Meteorology, 1979, 18(7) : 898-903. 